\title{
Evaluación de las condiciones de crecimiento celular para la producción de astaxantina a patir de la microalga Haematococcus pluvialis
}

\author{
Evaluation of Cell Growth Conditions for the Astaxanthin Production as \\ of Haematococcus pluvialis Microalgae
}

Clara Milena Niño Castillo ${ }^{1}$, Francis Carolina Rodríguez Rivera ${ }^{1}$, Luis Eduardo Díaz ${ }^{2}$, Ana Graciela Lancheros Díaz ${ }^{3}$

\begin{abstract}
Resumen
En la actualidad la astaxantina de origen natural es uno de los pigmentos carotenoides con importantes aplicaciones en la industria alimenticia, farmacéutica y cosmética, debido a sus grandes propiedades dentro de las que se destaca su gran poder antioxidante, efecto preventivo del cáncer, incremento de la respuesta inmune, inhibición de los radicales libres entre muchas otras. Haematococcus pluvialis es una microalga verde de agua dulce y es una de las fuentes naturales con mayor producción de astaxantina ya que es capaz de acumular hasta un 3\% de astaxantina en peso seco. El objetivo del presente trabajo fue determinar el medio de cultivo y las condiciones óptimas para el crecimiento y la producción de astaxantina a partir de Haematococcus pluvialis. La influencia de diferentes factores como el $\mathrm{pH}$, temperatura, agitación, aireación $\mathrm{CO}_{2}$ e iluminación favorecen el crecimiento celular, al darle un ambiente óptimo a la microalga.
\end{abstract}

Para determinar las condiciones nutricionales óptimas, se evaluó el efecto de diferentes medios de cultivo (BBM, OHM, RM) en Birreactores de $500 \mathrm{~mL}$ con $350 \mathrm{~mL}$ de medio y $1 \times 10^{4} \mathrm{cel} / \mathrm{ml}$ de inóculo en fase exponencial, las condiciones de cultivo empleadas fueron: $\mathrm{pH} .6 .7$ a 7, $\mathrm{CO}_{2}$ al 5\%, fotoperiodo de 16 horas luz 8 oscuridad, irradianza $70 \mu \mathrm{E} / \mathrm{m}^{2} \mathrm{~s}$; Los resultados mostraron que el mayor crecimiento o producción celular se obtuvo en el medio RM con 7,5 x 10 $\mathrm{cel} / \mathrm{ml}$ en el día 36, y la mayor producción de astaxantina se obtuvo en el medio RM con una concentración de $8.3 \mu \mathrm{g} / \mathrm{ml}$ en el tratamiento 4 .

Palabras claves: Haematococcus pluvialis, astaxantina, crecimiento celular, medios de cultivo.

\begin{abstract}
1. Bacteriólogas y Laboratoristas Clínicas con énfasis en Microbiología Industrial Universidad Colegio Mayor de Cundinamarca.

2. Docente Investigador Universidad de la Sabana.

3. Docente Investigadora Grupo Bioprocesos y Control, Universidad Colegio Mayor de Cundinamarca.

Correspondencia: aglancheros@unicolmayor.edu.co
\end{abstract}

Currently astaxanthin of natural origin is one of the carotenoids with important applications in the food, pharmaceutical and cosmetics, due to their large properties within its powerful antioxidant, cancer preventive effect, increase the immune response, inhibiting free radicals among many others. Haematococcus pluvialis is a freshwater green microalgae and is one of the largest natural sources of astaxanthin production as it is able to accumulate up to 3\% astaxanthin by dry weight. The aim of this study was to determine the culture medium and the optimal conditions for growth 
and production of astaxanthin from Haematococcus pluvialis. The influence of different factors such as $\mathrm{pH}$, temperature, agitation, aeration and lighting, $\mathrm{CO}_{2}$, promote cell growth, by giving an optimal environment to microalgae.

To determine the optimal nutritional conditions for cell growth the effect of different culture media (BBM, OHM, RM) in bioreactors of $500 \mathrm{ml}$ with $350 \mathrm{~mL}$ of medium and 1x104 cells $/ \mathrm{ml}$ inoculum was evaluated in exponential phase, the culture conditions employed they were: $\mathrm{pH}$. 6.7 to $7,5 \% \mathrm{CO}^{2}, 16$ hours photoperiod Light 8 dark, irradiance $70 \mu \mathrm{E} / \mathrm{m}^{2}$ s; The results showed that the highest growth and cell reproduction was obtained in the middle RM with $7.5 \times 10^{5}$ cells $/ \mathrm{ml}$ on day 36 , and increased production of astaxanthin was obtained in the middle RM with a concentration of $8.3 \mathrm{ug} / \mathrm{ml}$ in the treatment 4 .

Keywords: Haematococcus pluvialis, astaxanthin, cell growth, culture media.

\section{Introducción}

Los carotenoides tienen importantes funciones biológicas como precursores de vitamina A, estimulantes de la respuesta inmune, prevención del cáncer y poder antioxidante (1).

Los carotenoides son pigmentos naturales liposolubles, según su naturaleza química se dividen en dos grupos principales, carotenos y xantofilas. La astaxantina es un carotenoide de tipo xantofila que en los últimos años ha tomado gran importancia debido a su gran aplicabilidad en la industria alimenticia, farmacéutica y cosmética ya que aporta múltiples beneficios a la salud humana y animal por sus diversas propiedades, dentro de las que se destacan su efecto antiinflamatorio, anticancerígeno, preventivo contra los efectos de los rayos UV y su gran poder antioxidante, siendo 550 veces más potente que la vitamina $\mathrm{E}$ y 11 veces más que el betacaroteno. Además, en la industria alimenticia este metabolito secundario es utilizado para generar la pigmentación roja de algunos peces como salmón, trucha, camarón, y algunos peces ornamentales, crustáceos y aves (2). Los pigmentos carotenoides son de gran importancia para diferentes sectores, entre los que se destacan la acuicultura, la ornitología y la industria farmacéutica, desempeñando funciones vita- les en la fisiología y la salud de diferentes especies biológicas (3).

La principal limitación en la comercialización de la astaxantina de origen natural radica en la dificultad y el elevado costo de su síntesis lo que genera baja rentabilidad en comparación con la síntesis de astaxantina de forma sintética (4); $H$. pluvialis es una microalga verde de agua dulce y es una de las fuentes naturales con mayor producción de astaxantina ya que es capaz de acumular hasta un 3\% de astaxantina en peso seco (5). El cultivo y las extremadas condiciones de crecimiento de Haematococcus pluvialis dificulta la producción de astaxantina a nivel industrial ya que este presenta un crecimiento lento, baja concentración celular, alta susceptibilidad a daños hidrodinámicos y un ciclo de vida complejo con distintas formas celulares que varían de acuerdo con los factores del cultivo, ya que para lograr un adecuado crecimiento con una elevada producción de astaxantina es necesario que el cultivo se encuentre en condiciones de estrés adecuadas, como lo son la intensidad lumínica, temperatura, concentración de nitrógeno, fósforo, dióxido de carbono, minerales, entre otros (6). Por este motivo, el objetivo principal de esta investigación fue la determinación de un medio de cultivo óptimo. 
Kobayashi en 1992 (7) examinó el crecimiento de Haematococcus pluvialis en un medio basal de etilo que se encuentra constituido por sales, aminoácidos y acetato, sometiéndolo a condiciones heterotróficas y mixotróficas evaluando como factor principal la influencia de luz-oscuridad. El trabajo mostró que $H$. pluvialis es capaz de crecer y producir astaxantina tanto en condiciones mixotróficas (acetato-12h luz y $12 \mathrm{~h}$ oscuridad) como en condiciones heterotróficas (acetato-oscuridad), razón por la cual se llegó a la conclusión que $H$. pluvialis puede crecer y producir astaxantina en condiciones de luz y oscuridad siempre y cuando se haya adicionado acetato.

Tripathi en 1999 (8) evaluó cinco medios de cultivo en condición autotrófica, heterotrófica y mixotrófica con el fin de determinar el medio y la condición de crecimiento óptimas y que generen la máxima producción de astaxantina. Los medios de cultivos que se emplearon en condiciones autotróficas fueron el medio basal bold (BBM) y el medio Z8, en condiciones heterotróficas y el medio (KM1)) bajo una intensidad lumínica constante de 1.5 klux y a una temperatura de 25 $+/-1^{\circ} \mathrm{C}$, en condiciones mixotróficas. Los medios de cultivo se prepararon a partir de los medios de cultivo autótrofos (BBM) completándolo con acetato de sodio (MM1) y acetato de sodio con l-asparagina (MM2). Los resultados mostraron que de los medios sometidos a condición de autotrofía el medio BBM obtuvo la mayor tasa de crecimiento con un recuento de celular máximo de $1.5 \times 10^{5}$ células/ml en comparación con el medio Z8 el cual obtuvo un recuento celular de $0.8 \times 10^{5}$ células $/ \mathrm{ml}$, analizados a los 10 días. En condiciones heterotróficas el medio KM1 obtuvo un recuento considerablemente mayor a los 5 días con un recuento celular máximo de 4,35 × $10^{5}$ células/ $\mathrm{ml}, 20$ veces más que el recuento celular en el medio de BBM que obtuvo un valor de $0.217 \times 10^{5}$ al mismo día. Se pudo determinar que el medio Z8 es adecuado para el mantenimiento de los cultivos y el medio MM1 es adecuado para el mantenimiento de las células vegetativas.
Salazar en 2001 (9) estudio el efecto de la concentración de $\mathrm{NaNO} 3$ en el crecimiento de Haematococcus pluvialis para determinar condiciones óptimas del cultivo, utilizando tres cepas de H.pluvialis (J,A,G) cultivadas en medio Bristol con tres concentraciones de $\mathrm{NaNO} 3$ distintas $(0.25,0.75$, y $1.25 \mathrm{~g})$ y empleando condiciones de crecimiento específicas como agitación orbital, temperatura: $25^{\circ} \mathrm{C}$, intensidad de luz: $4850 \mathrm{~lx}$, en un fotoperiodo de $12 \mathrm{~h}$ de luz y $12 \mathrm{~h}$ de oscuridad; se logró demostrar que el mayor crecimiento de H.pluvialis se obtiene en los medios que contienen $1.25 \mathrm{~g} / \mathrm{l}$ en el día 45 en dos de las tres cepas estudiadas.

Kobayashi en 2001 (10) analizó las distribuciones del tamaño de las células quísticas y el contenido intracelular de astaxantina en $H$. Pluvialis, las cuales fueron aisladas por centrifugación de gradiente de densidad de sacarosa y se examinó la relación entre el tamańo de los quistes y el contenido de astaxantina, usando el diclorofluoresceno fluorescente (DCF). La formación de DCF en las células quísticas se redujo cuando había un mayor diámetro de la célula. Esta disminución también se correlacionó con el aumento en el contenido de astaxantina. Por lo tanto, el diámetro celular y el contenido fluorescente DCF de las células quísticas sería buen parámetro para seleccionar cepas astaxantina híper productivas de poblaciones nativas de $H$. pluvialis.

Fabregas en 2001 (11) realizó un sistema de cultivo en dos etapas para la producción de astaxantina a partir de $H$. pluvialis, las cepas fueron cultivadas en medio OHM con un $\mathrm{pH}$ 7,2 y 7,8 con una intensidad lumínica de 40 fotones mol m-2 s-1que fue proporcionada por lámparas fluorescentes de luz blanca regido por un ciclo de 12 horas luz: 12 horas oscuridad, a $25^{\circ} \mathrm{C}$, aireación intermitentemente durante 10 segundos, cada 10 min. a una velocidad de $250 \mathrm{ml} \mathrm{min.,} \mathrm{en} \mathrm{la} \mathrm{pri-}$ mera etapa se realizaron cultivos semi-continuos con tasas de renovación diaria entre 10 y $40 \%$ con el fin de lograr una obtención de biomasa 
significativa. En la segunda etapa, los cultivos fueron sometidos a altas intensidades de 240 fotones mol m-2 s-1 durante 15 días con el fin de estimular la transición a la etapa aplanospora y con esto conseguir la producción de astaxantina. Los resultados revelaron que todos los cultivos alcanzaron el estado estacionario de 2 a 5 días del cultivo semi-continuo obteniendo una densidad celular de $5,72 \times 10^{5}$ células $/ \mathrm{ml}$ y una renovación diaria de $10 \%$, además se detectó que los cultivos obtenidos a una tasa de renovación del 10\% después de 3 días de ser sometidos a una alta intensidad lumínica se obtuvieron valores de hasta $49 \mu \mathrm{g}$ $\mathrm{ml}^{-1}$ en el día 15; cuando las células se obtienen a una tasa de renovación del $40 \%$ se detectó que después de 9 días de ser sometida a intensidad lumínica el crecimiento celular cesa y el nitrógeno se agota y en ese momento se generan períodos de inducción de astaxantina; la productividad máxima se generó a los 15 días con un valor de 9,6 $\mathrm{mg} \mathrm{l}^{-1}$ día $^{-1}$ con una tasa de renovación del $40 \%$. (10)

Cifuentes en 2003 (12) evaluó diferentes condiciones del cultivo para optimizar el crecimiento de biomasa de $H$. pluvialis y con esto lograr la producción de astaxantina en condiciones autótrofas con la adición de $\mathrm{NaNO}_{3}, \mathrm{NH} 4 \mathrm{Cl}$ y urea $\mathrm{y}$ en condiciones mixotróficas con $4,8,12 \mathrm{mM}$ de acetato de sodio sometidas a densidad de flujo de fotones (PFD). La carotenogénesis fue inducida por 3 mecanismos: la adición de $\mathrm{NaCl}(0,2$ y 0,8\%), privación de nitrógeno y la alta PFD. Para el crecimiento inicial de Haematococcus pluvialis se utilizó Medio Bristol al cual se le ajustó el $\mathrm{pH}$ a 6. Esta acidificación condujo a aumentar el crecimiento exponencial específico del alga, la densidad inicial de biomasa fue $2.0 \times 10^{3}$ células $/ \mathrm{ml}$. Los resultados mostraron que la mejor fuente de nitrógeno fue el cloruro de amonio para la obtención de mayor cantidad de biomasa que produjo una densidad celular de $7.8 \times 10^{4}$ células $/ \mathrm{ml}$. La luz era el mejor factor inductivo de la carotenogénesis y la mayor producción de carotenoides se observó en los cultivos que fueron pre-cultivados en nitrato, de los cuales con poca luz se obtuvieron (22 pg. por célula) y el contenido de astaxantina por peso seco fue de 10,3 mg. Así mismo se demostró que el $\mathrm{NaCl}$ causó un aumento en el contenido de carotenoides por célula pero indujo a la mortalidad celular.

Domínguez en 2004 (13) determinó las condiciones ambientales como intensidad de luz, aireación en el crecimiento y producción de astaxantina por H. pluvialis. La cepa se inoculó en el medio de cultivo Bold Basal (BBM) conformado por sales (sulfatos, nitratos, fosfatos), elementos trazas y ácido bórico; en el medio de cultivo (BG-11) compuesto por sales nitratos, sulfatos, ácido cítrico, EDTA; en el medio (FAB) teniendo el mismo contenido del Bold Basal (BBM) sin elementos traza; en el medio (BAR) con los mismos componentes exceptuando los elementos traza, sin ácido cítrico y sin EDTA; el medio (BAR) compuesto por sales, EDTA, ácido cítrico, ácido bórico. El crecimiento de H.pluvialis, en los diferentes medios de cultivo y condiciones de cultivo dio como resultado la mayor tasa de crecimiento en el Medio Basal Bold (BBM) con iluminación continua y aireación, obteniendo un conteo de $34.67 \times 10^{4}$ cél/ $\mathrm{ml}$ con una diferencia significativa con respecto a los demás medios de cultivo evaluados; la mayor producción de astaxantina en los medios evaluados fue dada en el medio de cultivo (BAR) con $3.30 \times 10^{4} \mathrm{mg} / \mathrm{g}$ de biomasa. (12)

Imamoğlu en 2007 (14) investigó y comparó los efectos de dos medios de cultivo RM y BG11 y las condiciones de crecimiento para lograr maximizar la tasa de crecimiento de dos cepas de $H$. pluvialis (EGEMACC-35 y EGEMACC-36). Las cepas fueron cultivadas bajo condiciones autotróficas (presencia de luz y suministro de CO2.), mixotróficas (presencia de luz y suministro de acetato de sodio) y heterotróficas (presencia de oscuridad suministro de acetato de sodio) con una temperatura de $25^{\circ} \mathrm{C}$, aireación continua y una intensidad de luz de 75 mol-fotones. Teniendo en cuenta el crecimiento de las dos cepas 
estudiadas se logró determinar que el crecimiento para la cepa EGEMACC-35 se incrementó en un $90 \%$ con respecto a la cepa EGEMACC-36 en el modelo de cultivo bajo condiciones autótrofas. Se considera que la luz es esencial para el ciclo de vida de $H$. pluvialis y estudios previos sobre la nutrición de $H$. pluvialis lograron demostrar que el acetato es una importante fuente de carbono que mejora tanto el crecimiento como la carotenogénesis.

González en 2009 (15) planteó como objetivo de este estudio fue comparar el crecimiento y la producción de carotenoides totales entre cuatro cepas de $H$. pluvialis aisladas de diferentes micro hábitats en dos ubicaciones geográficas en Chile. El crecimiento se llevó a cabo en medio Bristol en condiciones autotróficas y mixotroficas (con adición de 2 y $10 \mathrm{mM}$ de acetato de sodio) a una temperatura de $22 \pm 1{ }^{\circ} \mathrm{C}$. y a dos densidades de flujo fotónico (DFF) $(20$ y $85 \mu \mathrm{mol} \mathrm{m}-2 \mathrm{~s}-1)$. El contenido de carotenoides totales se estimó el día 45.(14). Los resultados mostraron que la mayoría de las cepas crecieron mejor en condiciones autotróficas y las más altas tasas de crecimiento exponencial se presentaron a la mayor DFF en todas las cepas la cual fluctuó entre 0,73 y 1,20 divisiones/día a excepción de una de las 4 cepas que mostró la misma tasa de crecimiento exponencial que fue de 0,73 divisiones/día a cualquier DFF, además se comprobó que la densidad celular en el día 14 de las cepas CCM-UDEC 022 y CCM-UDEC 023 fue mayor cuando las cepas eran expuestas a la menor DFF con valores de 4,4 x $10^{5}$ y $8,9 \times 10^{5}$ cel./ $\mathrm{ml}$, respectivamente.

Hanan en 2013 (16) realizó un diseño factorial para detectar parámetros que puedan afectar el crecimiento de $H$. pluvialis evaluando variables como $\mathrm{pH}$, tamańo del inóculo, temperatura y presencia o ausencia de luz. El cultivo fue realizado en Medio BBM compuesto por 5 elementos con adición de acetato de sodio 0,25\% como fuente de carbono ya que este mejora el crecimiento y la carotenogénesis. Los resultados mostraron que el crecimiento experimental fue mayor de lo esperado: el crecimiento esperado era de 1,08 células $/ \mathrm{ml}$ y el crecimiento obtenido fue de 1.17 células $/ \mathrm{ml}$, por lo que se llegó a la conclusión que las condiciones para optimizar el crecimiento de $H$. pluvialis se obtienen utilizando un $\mathrm{pH}$ de 5 , un tamaño del inóculo de $40 \%$, temperatura de $30^{\circ} \mathrm{C}$ y este cultivo deberá estar en presencia de luz.

Nunes en 2013 (17) evaluó la respuesta celular de $H$. pluvialis bajo condiciones de estrés nutricional y la influencia de la luz para la inducción de la carotenogénesis. Las cepas fueron cultivadas en medio WC con pH 7,0, solución de hierro y EDTA y solución de vitaminas durante 15 días, con aireación atmosférica a una velocidad de $100 \mathrm{ml} /$ min, fotoperiodo de 12 horas y temperatura de $23^{\circ} \mathrm{C}$. Los cultivos se mantuvieron en una intensidad de luz de 50 micromoles de fotones tiempo necesario para que el cultivo llegara a la fase exponencial. En conclusión, con la adición de $\mathrm{CO}_{2}$ y el aumento de la iluminación se observó un aumento de la proporción de carotenoides/clorofila y biomasa celular optimizando la carotenogenesis en $H$. pluvialis y aumentando la producción de astaxantina.

\section{Materiales y métodos}

Este trabajo de investigación se basó en un estudio de tipo descriptivo y correlacional, en donde se logró comparar 3 medios de cultivo (RM, OHM y BBM), bajo condiciones de estrés para que se dé mayor cantidad de biomasa y por ende mayor producción de astaxantina a partir de la microalga Haematococcus pluvialis.

La población de este estudio fue la microalga Haematococcus pluvialis, obtenida a partir de una cepa suministrada por la Facultad de Ingeniería de la Universidad de la Sabana, la cual se trabajó por un tiempo de 4 meses y medio aproximadamente, con los medios de cultivo RM, BBM y OHM escogidos según el estudio bibliográfico con mejor producción de astaxantina. 
El tipo de muestra a usar fue el inóculo de Haematococcus pluvialis. Se trabajó por triplicado en los 3 medios de cultivo RM, BBM y OHM bajo las condiciones propuestas. Se consideró como unidad de análisis el crecimiento celular y la producción de astaxantina.

\section{Variables}

Variable dependiente: Crecimiento celular y la producción de astaxantina.

Variable independiente: Medios de cultivo, que generan las condiciones óptimas para crecimiento celular por parte de Haematococcus pluvialis.

\section{Técnicas y procedimientos}

Revisión bibliográfica: para definir la metodología a utilizar se realizó una revisión bibliográfica teniendo en cuenta los medios de cultivo, condiciones de crecimiento como $\mathrm{pH}$, temperatura, fotoperíodo, etc. (Datos no mostrados).

\section{Microorganismo}

La microalga Haematococcus pluvialis UTEX, cepa de referencia (colección de cultivos de algas, Universidad de Texas, Austin TX,USA) suministrada por la Facultad de Ingeniería de la Universidad de la Sabana.

\section{Preparación del preinóculo}

La preparación del preinóculo se realizó a partir de la cepa de referencia, la cual se transfirió a un Erlenmeyer de 3L con adición de 2L de medio de cultivo MES Volvox estéril con ajuste de $\mathrm{pH}$ de 6.7, fotoperiodo de 18 horas luz -6 horas de oscuridad, $\mathrm{CO}_{2} 5 \%$, agitación manual 10 segundos una vez al día a $25^{\circ} \mathrm{C}$ por 21 días (Ver Figura 1).

Se realizó monitoreo del preinóculo mediante microscopia donde se evaluó el color, morfología y viabilidad celular; se hizo conteo celular mediante cámara de Neubauer obteniendo una concentración celular de $16 \times 10^{4}$ células $/ \mathrm{ml}$; a partir de esta concentración se procedió a determinar el volumen de inóculo a usar por birreactor para obtener una concentración inicial de $1 \times 10^{4}$ en un volumen de $350 \mathrm{ml}$ de medio de cultivo, mediante la siguiente fórmula: $\mathrm{V} 1 \mathrm{C} 1=\mathrm{V} 2 \mathrm{C} 2$.

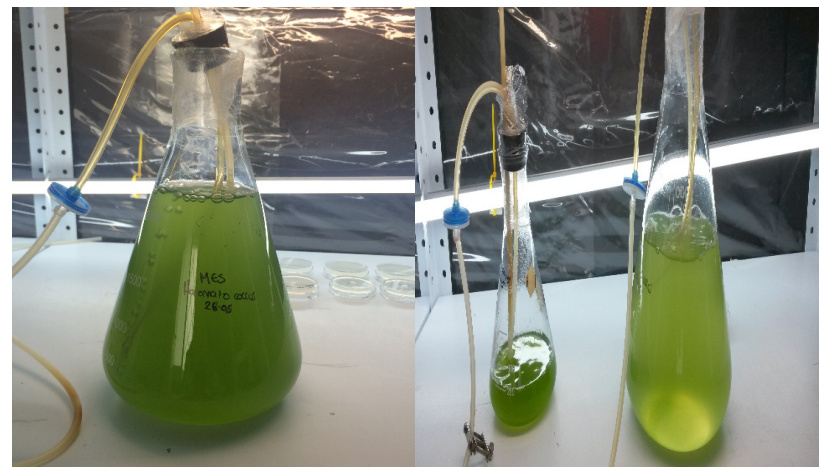

Figura 1. Preinóculo de la microalga Haematococcus pluvialis.

\section{Evaluación de los medios de cultivo}

Con el fin de obtener la mejor productividad tanto de biomasa como de astaxantina y basándose en la revisión bibliográfica (Tabla 1), se evaluaron 3 medios de cultivo: BBM, RM y OHM, los cuales mostraron los mejores resultados.

\section{Medios de cultivo}

El cultivo de $H$. pluvialis se realizó en biorreactores de $500 \mathrm{ml}$ con adición de $350 \mathrm{ml}$ de cada uno de los medios a evaluar, RM, BBM, OHM (ver Tabla 1), por triplicado.

Tabla 1. Medios de cultivo.

\begin{tabular}{|c|c|c|c|c|c|}
\hline Medio RM1 & $\mathrm{mg} / \mathrm{l}$ & $\begin{array}{l}\text { Medio } \\
\text { BBM2 }\end{array}$ & $\mathrm{mg} / \mathrm{l}$ & Medio OHM3 & $\mathrm{mg} / \mathrm{l}$ \\
\hline $\mathrm{NaNo}_{3}$ & 300 & $\mathrm{KH}_{2} \mathrm{PO}_{4}$ & 175 & $\mathrm{KNO}_{3}$ & 410 \\
\hline $\mathrm{K}_{2} \mathrm{HPO}_{4}$ & 80 & $\mathrm{CaCl}_{2}$ & 25 & $\mathrm{Na}_{2} \mathrm{HPO}_{4}$ & 30 \\
\hline $\mathrm{KH}_{2} \mathrm{PO}_{4}$ & 20 & $\mathrm{MgSO}_{4}$ & 75 & $\mathrm{MgSO}_{4} \cdot 7 \mathrm{H}_{2} \mathrm{O}$ & 246.5 \\
\hline $\mathrm{MgSO}_{4} 7 \mathrm{H}_{2} \mathrm{O}$ & 10 & $\mathrm{NaNO}_{3}$ & 250 & $\mathrm{Cl}_{2} \mathrm{Ca} \cdot 2 \mathrm{H}_{2} \mathrm{O}$ & 110.9 \\
\hline $\mathrm{CaCl}_{2} \cdot 2 \mathrm{H}_{2} \mathrm{O}$ & 58.5 & $\mathrm{~K}_{2} \mathrm{HPO}_{4}$ & 75 & $\mathrm{FeC}_{6} \mathrm{H}_{5} \mathrm{O}_{7} \cdot 5 \mathrm{H}_{2} \mathrm{O}$ & 2.62 \\
\hline EDTA & 7.5 & $\mathrm{NaCl}$ & 25 & $\mathrm{Cl}_{2} \mathrm{Co} .6 \mathrm{H}_{2} \mathrm{O}$ & 0.011 \\
\hline $\mathrm{NaCl}$ & 20 & $\mathrm{H}_{3} \mathrm{BO}_{3}$ & 11.42 & $\mathrm{CuSO}_{4} 5 \mathrm{H}_{2} \mathrm{O}$ & 0.012 \\
\hline $\mathrm{H}_{3} \mathrm{BO}_{3}$ & 0.3 & $\mathrm{ZnSO}_{4}$ & $8.82 \mathrm{~g} / \mathrm{L}$ & $\mathrm{Cr}_{2} \mathrm{O}_{3}$ & 0.076 \\
\hline $\mathrm{MnSO}_{4} \mathrm{H}_{2} \mathrm{O}$ & 1.5 & $\mathrm{MnCl}_{2}$ & $1.44 \mathrm{~g} / \mathrm{L}$ & $\mathrm{MnCl}_{2} \cdot 4 \mathrm{H}_{2} \mathrm{O}$ & 0.989 \\
\hline $\mathrm{ZnSO}_{4} \cdot 7 \mathrm{H}_{2} \mathrm{O}$ & 0.1 & $\mathrm{MoO}_{3}$ & $0.71 \mathrm{~g} / \mathrm{L}$ & $\mathrm{Na}_{2} \mathrm{MoO}_{4} \cdot 2 \mathrm{H}_{2} \mathrm{O}$ & 0.12 \\
\hline$\left(\mathrm{NH}_{4}\right) 6 \mathrm{MoO}_{4} \cdot 2 \mathrm{H}_{2} \mathrm{O}$ & 0.3 & $\mathrm{CuSO}_{4}$ & $1.57 \mathrm{~g} / \mathrm{L}$ & $\mathrm{Se}$ & 0.025 \\
\hline $\mathrm{CuSO}_{4} \cdot 5 \mathrm{H}_{2} \mathrm{O}$ & 0.08 & $\mathrm{Co}\left(\mathrm{NO}_{3}\right)_{2}$ & $0.49 \mathrm{~g} / \mathrm{L}$ & Biotina & $5 \mathrm{ml}$ \\
\hline $\mathrm{Co}\left(\mathrm{No}_{3}\right)_{2} \cdot 6 \mathrm{H}_{2} \mathrm{O}$ & 0.26 & $\mathrm{Na}_{2}$ EDTA & $50 \mathrm{~g} / \mathrm{L}$ & Tiamina & $5 \mathrm{ml}$ \\
\hline $\mathrm{FeCl}_{3} \cdot 6 \mathrm{H}_{2} \mathrm{O}$ & 17 & $\mathrm{KOH}$ & $3.1 \mathrm{~g} / \mathrm{L}$ & B12 & $5 \mathrm{ml}$ \\
\hline
\end{tabular}

1.(13). - 2.(20). - 3.(11) 
Para cumplir con los objetivos del ensayo, el estudio se centró en la obtención de la mayor concentración celular en donde se evaluaron tres medios de cultivo (BBM, OHM, RM) cada uno por triplicado para un total de 9 ensayos, utilizando un inóculo inicial de $1 \times 10^{4}$ células $/ \mathrm{ml}$ en Erlenmeyer de $500 \mathrm{ml}$ con un volumen de cultivo de $350 \mathrm{ml}$ en forma aséptica y sellándolos para garantizar esterilidad.

Todos los ensayos se realizaron bajo las siguientes condiciones:

- Ajuste de cada medio a un pH 6,7, fotoperiodo de $18 \mathrm{~h} \mathrm{luz}-6$ oscuridad, 70 microestein de irradianza con lámparas de luz fluorescentes blanca (Tlt20w/54RS, marca Philips) controlados a través de medidor de irradianza (biosphericalinstrumentsQSPL2101), temperatura $25^{\circ} \mathrm{c}$, agitación manual una vez al día durante 10 segundos, $\mathrm{CO}_{2} 5 \%$ a través de pipeta de $\mathrm{CO}_{2}$ suministrada con compresor para regular la cantidad de flujo.

- Se tomó $1 \mathrm{ml}$ de muestra para evaluar crecimiento celular y morfología cada tercer día, realizando conteo celular en cámara de Neubauer, evaluación de morfología celular por microscopia y registro fotográfico.

- Toma de muestra de $10 \mathrm{ml}$ para evolución de clorofila y astaxantina cada 6 días.

- Se realizó control de temperatura, $\mathrm{pH}$, agitación, fotoperiodo, irradianza y $\mathrm{CO}_{2}$.

Esta fase del estudio se evaluó durante 36 días.

\section{Curva de crecimiento}

Se realizó la curva de crecimiento a partir del número de células por ml cuantificados en cámara de
Neubauer durante 21 días obteniendo un total de 7 datos obtenidos por cada medio de cultivo.

\section{Cambios morfológicos celulares}

Los cambios de morfológicos se determinaron durante las diferentes fases de crecimiento de $H$. pluvialis y se documentaron por microfotografía.

\section{Ajuste de datos curvas de crecimiento celular}

A cada medio de cultivo se le realizó un ajuste de crecimiento celular según el modelo logístico empleando software DMfit basado en el modelo de Baranyi and Roberts (1994) del consorcio combase y compañía.

\section{Análisis estadístico de los datos}

Se realizó un análisis de varianza (ANOVA) de un factor (crecimiento celular) para los tres tratamientos (medios de cultivo) por triplicado con un nivel de confianza del 95\%, para este análisis se emplearon los software de Excel y Statplus.

\section{Resultados}

\section{Crecimiento celular}

En esta etapa de la investigación se evaluó el efecto de tres medios de cultivo (OHM, BBM y RM), los cuales fueron previamente seleccionados al hacer una revisión bibliográfica sobre la efectividad de los medios en el crecimiento celular de H. Pluvialis.

Se desarrolló un seguimiento fotográfico durante la fase de crecimiento para observar cambio en la morfología celular en cada uno de los medios evaluados. En la Figura 2 se registran los cambios en los días 0, 18, 24 y 36 del estudio.

\begin{tabular}{|l|l|l|l|}
\hline DÍA & OHM & BBM & RM \\
\hline 0 & & & \\
& & & \\
& & & \\
\hline
\end{tabular}




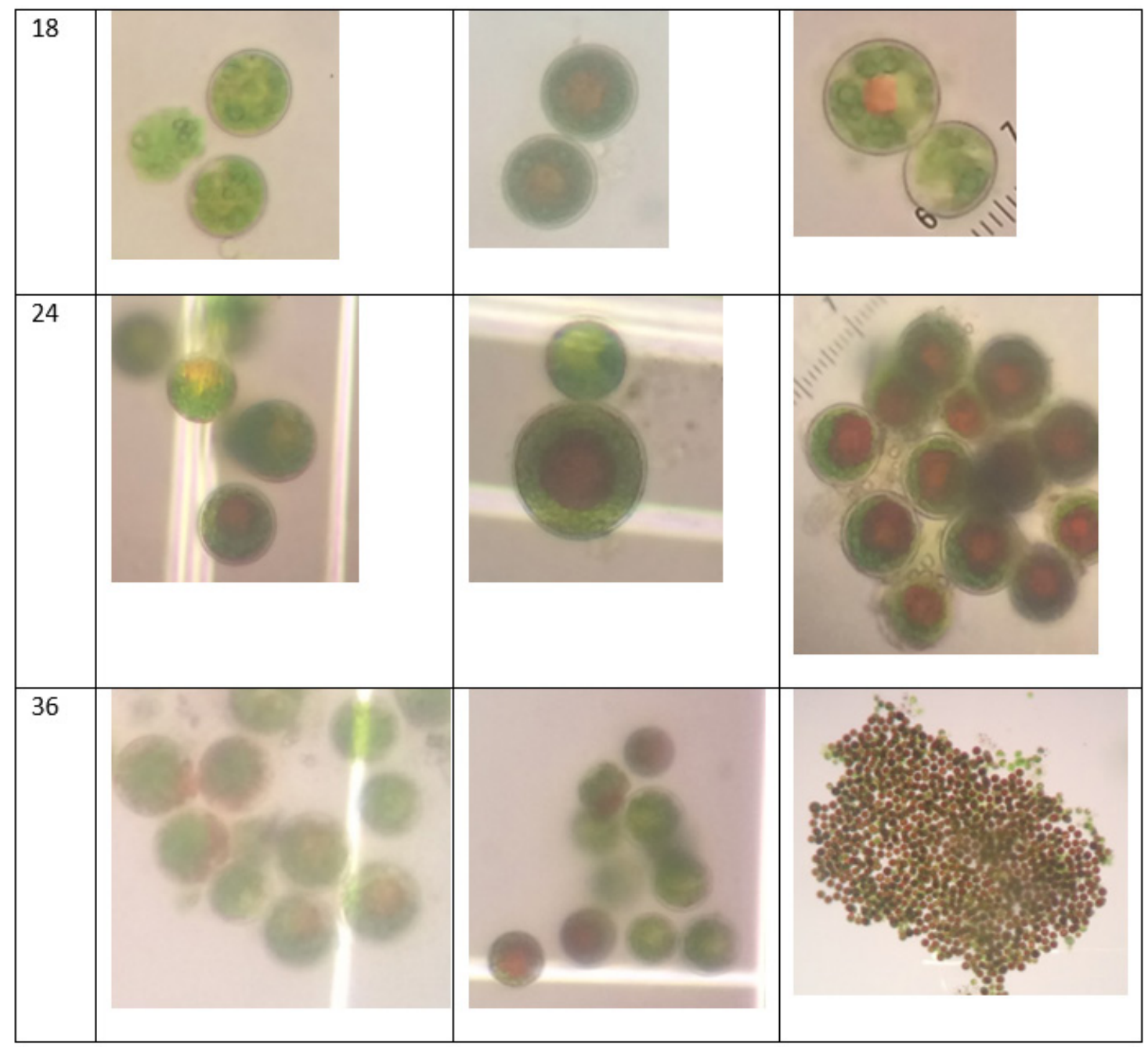

Figura 2. Cambios morfológicos en los medios de cultivo estudiados (OHM, BBM, RM). Fotos tomadas por las autoras de este proyecto.

A la vez se hizo registro fotográfico a los cambios macroscópicos celulares observables en los medios de cultivo (OHM, BBM, RM) en los biorreactores. En la Figura 3 se presentan los días 0, 15, 21 y 36 como los más representativos.

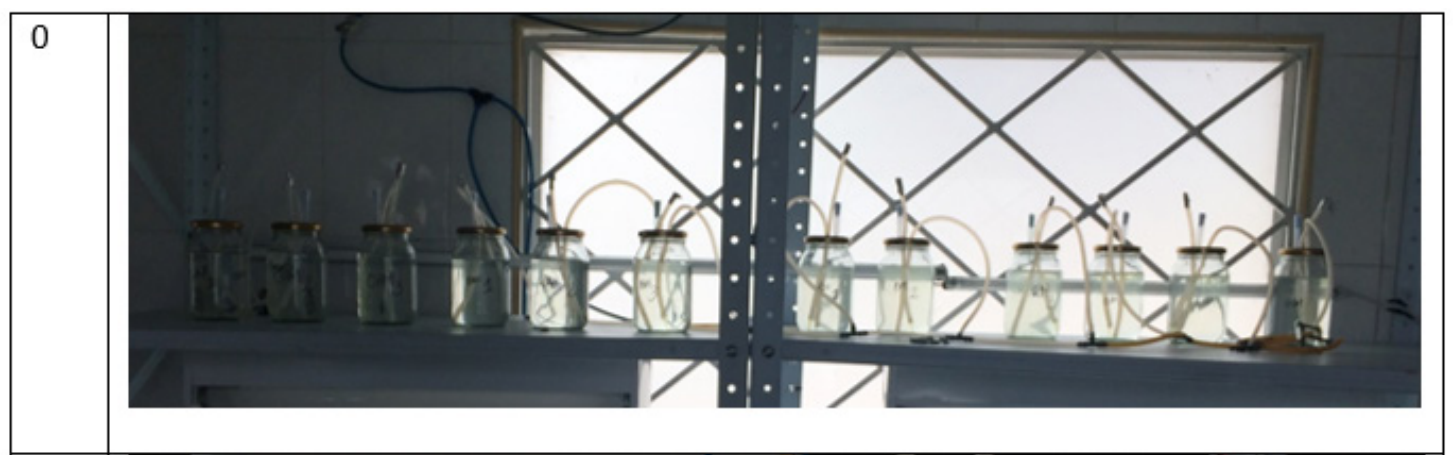




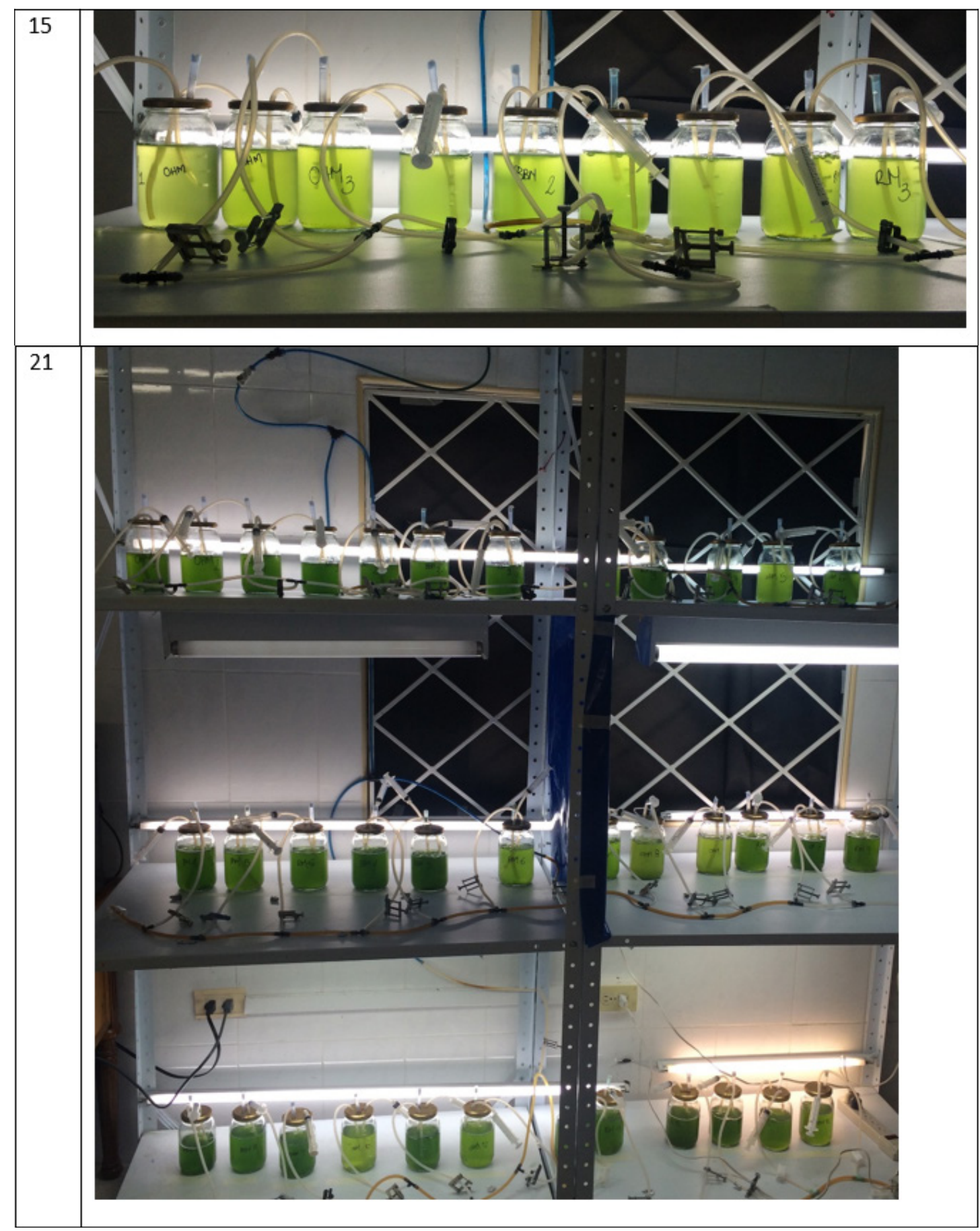




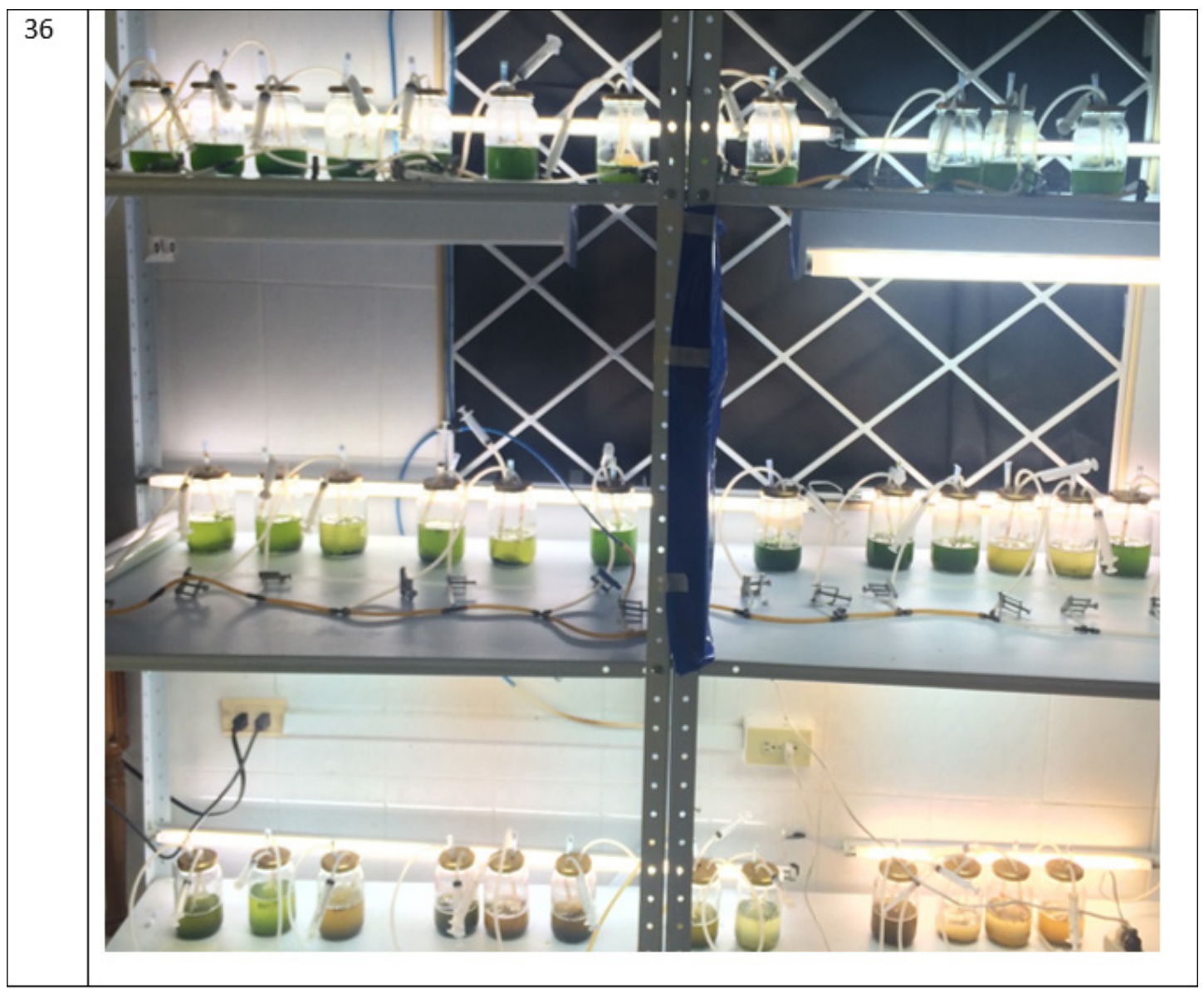

Figura 3. Cambios macroscópicos celulares en los medios de cultivo estudiados (OHM, BBM, RM). Fotos tomadas por las autoras de este proyecto.

Los datos de crecimiento se obtuvieron mediante recuento celular por cámara de Neubauer, con los cuales se construyó una cinética de crecimiento. Se observó que el medio de cultivo con mayor tasa de crecimiento fue el medio de cultivo RM, con un recuento celular de $7.55 \times 10^{5}$ células $/ \mathrm{ml}$, seguido del medio de cultivo BBM con un recuento de $3.88 \times 10^{5}$ células $/ \mathrm{ml}$, y por último, el medio de cultivo OHM con un recuento de $3.43 \times 10^{5}$ células/ml (ver figura 4 ).

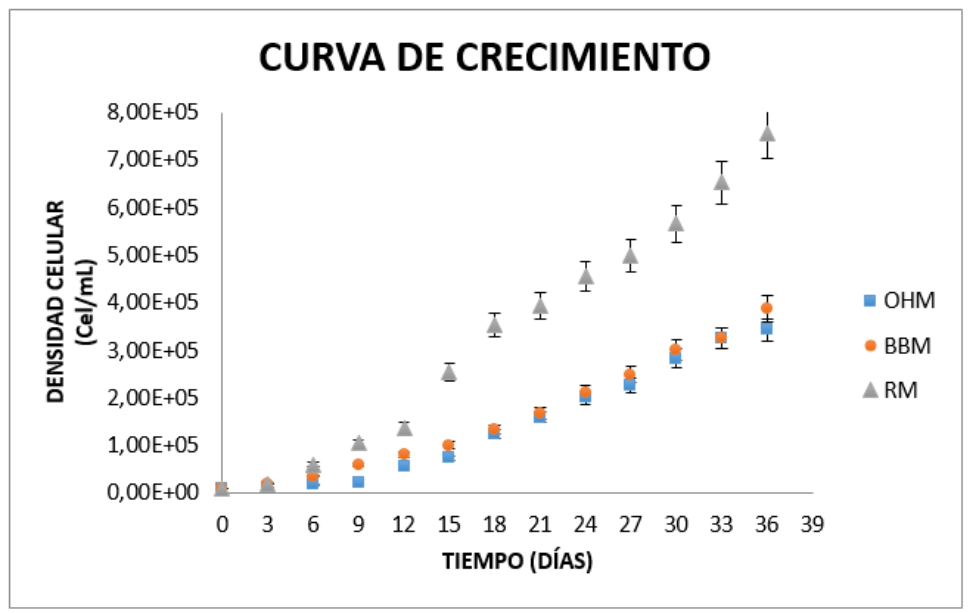

Figura 4. Curvas de crecimiento de la microalga Haematococcus pluvialis. 
Las tendencias de crecimiento fueron ajustadas al modelo logístico mediante transformación de los valores de Y, a los cuales se les calculó su logaritmo en base 10 para obtener log vs t del crecimiento celular. La idea de realizar el ajuste fue la de determinar si existían diferencias significativas en la velocidad de crecimiento de los medios probados. El medio de cultivo con la mayor velocidad específica de crecimiento fue RM con una velocidad específica de crecimiento de 0,092 células/ día (ver Tabla 2).

Tabla 2. Matriz de coeficientes del modelo logístico ajustado para los diferentes medios de cultivo.

\begin{tabular}{|r|r|r|r|r|r|}
\hline $\begin{array}{r}\text { Medio } \\
\text { de } \\
\text { cultivo }\end{array}$ & $\begin{array}{r}\text { No } \\
\text { Datos }\end{array}$ & $\begin{array}{r}\text { Dato } \\
\text { Mínimo }\end{array}$ & $\begin{array}{r}\text { Dato } \\
\text { Máximo }\end{array}$ & $\begin{array}{r}\text { Velocidad de } \\
\text { crecimiento }\end{array}$ & $\begin{array}{r}\text { Coeficiente de } \\
\text { determinación }\end{array}$ \\
\hline RM & 13 & 4 & 5,878 & 0,092 & $97 \%$ \\
\hline OHM & 13 & 4 & 5,535 & 0,062 & $99 \%$ \\
\hline BBM & 13 & 4 & 5,588 & 0,056 & $97 \%$ \\
\hline
\end{tabular}

Fuente: Autoras

Se realizó un análisis de varianza (ANOVA) de un factor (crecimiento celular) para los tres medios de cultivo (OHM, BBM, RM) del promedio de las réplicas, encontrando que para una confianza del 95\% existe diferencias estadísticas significativas $(\mathrm{p}=0,02)$ entre los diferentes medios de cultivo evaluados (ver Tabla 3).

Tabla 3. Análisis de varianza.

ANOVA
\begin{tabular}{r|r|r|r|r|r|r|}
\hline $\begin{array}{r}\text { Origen de la } \\
\text { Variación }\end{array}$ & Ss & df & MS & F & nivel p & F crit \\
\hline $\begin{array}{r}\text { Entre } \\
\text { Grupos }\end{array}$ & 42,97 & 8 & 5,37 & 3,57 & 0,004 & 2,21 \\
\hline $\begin{array}{r}\text { Dentro de } \\
\text { Grupos }\end{array}$ & 54,16 & 36 & 1,5 & & & \\
\hline Total & 97,13 & 44 & & & & \\
\hline
\end{tabular}

\section{Discusión}

Las microalgas tienen un elevado valor industrial gracias a su gran aplicabilidad, debido a que son útiles en la producción de biodiesel, el tratamiento de aguas residuales y la producción de proteína para la alimentación animal y humana, entre muchas otras propiedades. Además de las aplicaciones anteriores, estos microorganismos, tienen una característica especial como lo es la capacidad de acumular metabolitos de interés biotecnológico como los ácidos grasos poli-insaturados de cadena larga y los carotenoides (19).

Varios autores han realizado experimentos con el fin de obtener las mayores tasas de crecimiento de Haematococcus pluvialis ensayando diversos métodos, como lo son evaluación de $\mathrm{pH}$, temperatura óptima, agitación, $\mathrm{CO}_{2}$, exposición a una luz determinada por un tiempo, factores de estrés como adición de sales, aumento a la intensidad de luz (irradianza), entre otros más (20)(10)(13). Dentro de los requerimientos nutricionales para la producción de biomasa y astaxantina se encuentran los micronutrientes esenciales como el sodio, potasio, amonio, calcio, magnesio, hierro, cloro, azufre y los macronutrientes como carbono, hidrógeno, oxígeno y fósforo, que son de origen inorgánico y para los que se necesitan concentraciones entre $1-100 \mathrm{mg} / \mathrm{L}$, además se necesitan de elementos traza como magnesio, cobalto, níquel, vanadio, boro, cobre, zinc y molibdeno en concentraciones menores a $1 \mathrm{mg} / \mathrm{L}$, al igual que vitaminas y aminoácidos (21)(22)(23)(24).

El uso de diferentes medios de cultivo para optimizar el crecimiento de Haematococcus pluvialis ha sido uno de los grandes enfoques de la mayoría de los autores. En (13) se ensayó el Medio Basal Bold (BBM) obteniendo recuentos celulares de $3.5 \times 10^{5}$ células/ml. También en (7) se evaluó el crecimiento celular bajo condiciones normales en medio BBM obteniendo un bajo crecimiento celular $2 \times 10^{4}$ células $/ \mathrm{ml}$. Estos resultados se pueden comparar con los obtenidos en esta investigación con un crecimiento celular de $3.8 \times 10^{5}$ células $/ \mathrm{ml}$, los cuales presentan un aumento significativo con respecto a los obtenidos por Kobayashi (5), pero un crecimiento ligero comparado con el de Domínguez-Bocanegra (13). Asimismo, los datos de crecimiento celular obtenidos en el medio de cul- 


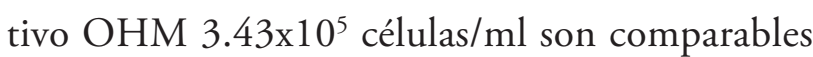
por los reportados por (11) con un crecimiento de $3.77 \times 10^{5} \mathrm{cel} / \mathrm{ml}$, bajo condiciones de cultivo similares, como fueron $\mathrm{pH}$ entre 6.2 y 7.4 , irradianza de 40 a $70 \mu \mathrm{Em}-2 \mathrm{~s}-1$ con lámparas de luz blanca.

El medio de cultivo RM en el presente trabajo obtuvo los mejores resultados alcanzando una concentración celular de $7.55 \times 10^{5}$ células $/ \mathrm{ml}$, lo que concuerda con lo reportado en la literatura y que lo considera como un medio de cultivo que alcanza concentraciones celulares más elevadas como las reportadas por (14) que alcanzó una concentración celular de $9.50 \times 10^{5}$ células $/ \mathrm{ml}$. Se debe tener en cuenta que los factores que pueden influir en el crecimiento celular como el $\mathrm{pH}$, la intensidad de la luz (24-28), aireación temperatura, agitación, suplementos entre otras pudieron influir en obtener una concentración menor a la ya reportada.

Otro factor a discutir es el método de recuento celular usado para el presente trabajo que aunque es uno de los métodos más usados por la mayoría de autores no es el método más específico ni preciso. Por este motivo se hace necesario el uso de métodos más exactos como la utilización de cell counting como lo reporta (15).

El medio de cultivo RM además de ser el que obtuvo las mejores tasas de crecimiento celular también es el mejor medio para la aplicación de factores de estrés con una mayor producción de astaxantina, datos no relacionados en este trabajo.

\section{Conclusiones}

La influencia de diferentes factores como el $\mathrm{pH}$, temperatura, agitación, aireación, $\mathrm{CO}_{2}$, e iluminación, favorecen el crecimiento celular de Haematococcus pluvialis, al darle un ambiente óptimo a la microalga en los medios de cultivo.

El medio de cultivo con mayor tasa de crecimiento, fue el medio de cultivo RM, con un recuento celular de $7.55 \times 10^{5}$ células $/ \mathrm{ml}$, seguido del medio de cultivo BBM con un recuento de $3.88 \times 10^{5}$ células/ml, y por último, el medio de cultivo OHM con un recuento de $3.43 \times 10^{5}$ células $/ \mathrm{ml}$.

El medio de cultivo RM por su composición nutricional favorece el crecimiento celular y que haya una mayor acumulación de astaxantina.

\section{Agradecimientos}

Un agradecimiento especial a Jorge Alberto Rodríguez Orjuela, técnico de los laboratorios de la Facultad de Ingeniería de la Universidad de la Sabana.

\section{Referencias}

1. López P. Efecto del consumo de Astaxantina en la salud. España: Universitat Oberta de Catalunya; 2012.

2. Lorenz RT, Cysewski GR. Commercial potential for Haematococcus microalgae as a natural source of astaxanthin. Trends Biotechnol. 2000;18(4):160-7.

3. Mauricio D, Landínez R. Evaluación del crecimiento y producción de astaxantina por Haematococcus pluvialis en un fotobiorreactor tipo airlift [tesis]. Bogotá: Universidad nacional de Colombia; 2013.

4. Dominguez A. Comparación de la producción de pigmentos carotenoides por Haematococcus pluvialis y Phaffia rhodozyma. España: Editorial Academica Española; 2011.

5. Kobayashi M, Kakizono T, Nagai S. Enhanced carotenoid biosynthesis by oxidative stress in acetate-induced cyst cells of a green unicellular alga, Haematococcus pluvialis. Appl Environ Microbiol. 1993; 59(3):867-73.

6. Lorenz R. A technical review of Haematococcus algae. Naturose Tech Bull. 1999.

7. Kobayashi M, Kakizono T, Yamaguchi K, Nishio N, Nagai S. Growth and astaxanthin formation of Haematococcus pluvialis in heterotrophic and mixotrophic conditions. J Ferment Bioeng. 1992; 74(1):17-20.

8. Tripathi U, Sarada R, Ramachandra Rao S, Ravishankar G a. Production of astaxanthin in Haematacoccus pluvialis cultured in various media. Bioresour Technol. 1999; 68(2):197-9.

9. Salazar M, Monroy O, Beristain R, Cuevas F, Mendoza C. Influencia del medio de cultivo en el crecimiento de Haematococcus pluvialis. IX Congreso Nacional de Biotecnología y Bioingeniería. México; 2012. 
10. Kobayashi M, Katsuragi T, Tani Y. Enlarged and astaxanthin-accumulating cyst cells of the green alga Haematococcus pluvialis. J Biosci Bioeng. 2001; 92(6):565-8.

11. Fábregas J, Otero A, Maseda A, Domínguez A. Two-stage cultures for the production of astaxanthin from Haematococcus pluvialis. J Biotechnol. 2001; 89(1):65-71.

12. Cifuentes AS, González M a., Vargas S, Hoeneisen M, González N. Optimization of biomass, total carotenoids and astaxanthin production in Haematococcus pluvialis Flotow strain Steptoe (Nevada, USA) under laboratory conditions. Biol Res. 2003; 36(3-4):343-57.

13. Dominguez-Bocanegra A. R., Legarreta I. G., Jerónimo F. M.,Campocosio, A. T. Influence of environmental and nutritional factors in the production of astaxanthin from Haematococcus pluvialis. Bioresource technology 2004; 92(2), 209-214.

14. Imamoglu E, Sukan FV. Effect of Different Culture Media and Light Intensities on Growth of Haematococcus pluvialis. International journal of natural and engineering sciences 2007; 1(3):5-9

15. González M a, Cifuentes AS, Gómez PI. Growth and Total Carotenoid Content in Four Chilean Strains of Haematococcus Pluvialis Flotow, Under Laboratory Conditions. Gayana Botánica. 2009; 66(1):58-70.

16. Hanan N, Al-Shorgani N, Shukor H, Rahman N, Kalil M. Pre-optimization conditions for Haematococcus pluvialis growth. Int J Adv Sci Eng Inf Technol. 2013; 3(2):70-3.

17. Nunes M., Vieira A, Pinto E, Carneiro R, Monteiro A. Carotenogênese em células de Haematococcus pluvialis induzidas pelos estresses luminoso e nutricional. Pesquisa Agropecuária Brasileira 2013; 48(8): 825-832.

18. Baranyi J, Roberts T. A dynamic approach to predicting bacterial growth in food. Int J Food Microbiol 1994; nov: 23(34):277-94.

19. Cardozo KHM, Guaratini T, Barros MP, Falcão VR, Tonon AP, Lopez NP, et al. Metabolites from algae with economical impact. Comp Biochem Physiol - C Toxicol Pharmacol. 2007; 146(1-2 SPEC. ISS.): 60-78.

20. Li L, Song Z, Zhan Y, Duan S, Zhao Q, Liu Y. Effect of Vitamin-B 12 and Vitamin-H on the Growth and Astaxanthin Content of Haematococcus pluvialis Food Technol 2013;5(9):1139-42.

21. Steinbrenner J, Linden H. Regulation of Two Carotenoid Biosynthesis Genes Coding for Phytoene Synthase and Carotenoid Hydroxylase during Stress-Induced Astaxanthin Formation in the Green Alga Haematococcus pluvialis. plant physiol. 2001;125.

22. Lababpour A, Shimahara K, Hada K, Kyoui Y, Katsuda T, Katoh S. Fed-batch culture under illumination with blue light emitting diodes (LEDs) for astaxanthin production by Haematococcus pluvialis. J Biosci Bioeng. 2005;100(3):339-42.

23. Yoshimura S, Ranjbar R, Inoue R, Katsuda T, Katoh S. Effective utilization of transmitted light for astaxanthin production by Haematococcus pluvialis. J Biosci Bioeng. 2006;102(2):97101.

24. Ranjbar R, Inoue R, Katsuda T, Yamaji H, Katoh S. High efficiency production of astaxanthin in an airlift photobioreactor. J Biosci Bioeng. 2008; 106(2):204-7.

25. Camacho Kurmen Judith Elena, González Gloria, Klotz Bernadette. Producción de Astaxantina en Haematococcus pluvialis bajo diferentes condiciones de estrés. Nova [Internet]. 2013 Jan [cited 2017 marzo 11] ; 11( 19 ): 94-104. Available from: http://www.scielo.org.co/scielo.php?script=sci_arttext\&pid=S1794-24702013000100009\&lng=en.

26. Ramírez, L. C. C., et al. Solubilización de fosfatos: una función microbiana importante en el desarrollo vegetal. Nova. 2014; 12(21).

27. Galvez, Z. Y. A. and V. E. M. Burbano. Bacillus: género bacteriano que demuestra ser un importante solubilizador de fosfato. NOVA Publicación en Ciencias Biomédicas. 2015; 12(22): 165-177.

28. Rodríguez, O. E., Andrade, W. A., Díaz, F. E., \& Moncada, B. Actividad antimicrobiana de líquenes de la cuenca alta del rio Bogotá. 2015; Nova, 13(23). 